\title{
VARIOUS TECHNIQUES ENHANCING BIOAVAILABILTY OF POORLY WATER SOLUBLE DRUGS
}

\author{
Thakur Anil Kumar*, Nirmala, Harikumar S L \\ Rayat and Bahra Institute of Phamacy, Sahauran, Kharar, District Mohali, Punjab, India-140104 \\ *Corresponding Author's E-mail: anilkatwal63@gmail.com, Contact No: +91-8699152639
}

\begin{abstract}
A drug administered in solution form immediately available for absorption and efficiently absorbed than the same amount of drug administered in a tablet or capsule form. Solubility is a most important parameter for the oral bioavailability of poorly soluble drugs. Dissolution of drug is the rate determining step for oral absorption of the poorly water soluble drugs, which can subsequently affect the in vivo absorption of drug. Currently only $8 \%$ of new drug candidates have both high solubility and permeability. Because of solubility problem of many drugs the bioavailability of them gets affected and hence solubility enhancement becomes necessary. It is now possible that to increase the solubility of poorly soluble drugs with the help of various techniques such as Physical method,Chemical method. Co-crystallisation, co-solvency solubilizing agents, molecular encapsulation with cyclodextrins,nanotechnology approaches and hydrotropy.

Key-Words: Solubility, Dissolution and Bioavailability
\end{abstract}

\section{INTRODUCTION}

Orally administered drugs completely absorb only when they show fair solubility in gastric medium and such drugs shows good bioavailability. Recently more than $40 \%$ NCEs (new chemical entities) developed in Pharmaceutical Industry are practically insoluble in water. These poorly water soluble drugs are allied with slow drug absorption leading to inadequate and variable bioavailability and gastrointestinal mucosal toxicity ${ }^{1}$.

The goal of drug delivery system is to achieve blood plasma drug concentration. There are different methods used for the enhancement of the drug absorption. A drug injected intravascular (intravenously and intra- arterially) directly enters the systemic circulation and exerts its pharmacologic effects. However, most of the drugs are administered extravascularly, generally orally. As the oral drug delivery is the simplest and easiest way of drug administration, because of the greater stability, lesser bulk, accurate dosage, cheaper cost of production and easy process, solid oral dosage forms have advantages over other dosage forms ${ }^{2,3}$.

Therapeutic effectiveness of a drug depends upon the bioavailability and ultimately upon the solubility of drug molecules. Solubility is one of the important parameter to achieve desired concentration of drug in systemic circulation for pharmacological response to be shown. Currently only $8 \%$ of new drug candidates have both high solubility and permeability ${ }^{4}$.

\section{FACTORS AFFECTING SOLUBILITY}

The solubility depends on the physical form of the solid, the nature and composition of solvent medium as well as temperature and pressure of system ${ }^{5}$.

\section{- Particle size}

The size of the solid particle influences the solubility because as a particle becomes smaller, the surface area to volume ratio increases. The larger surface area allows a greater interaction with the solvent. The effect of particle size on solubility can be explained as per the following equation ${ }^{6}$.

Where, $\mathbf{S}$ is the solubility of infinitely large particles, $\mathbf{S}$ is the solubility of fine particles, $\mathbf{V}$ is molar volume, $\boldsymbol{G}$ is the surface tension of the solid, $\mathbf{R}$ is the radius of the fine particle

$$
\log \frac{S}{S_{0}}=\frac{2 \gamma V}{2.303 \text { R }}
$$

\section{- Temperature}

Temperature will affect solubility. If the solution process absorbs energy then the solubility will be increased as the temperature is increased. If the solution process releases energy then the solubility will decrease with increasing temperature ${ }^{7}$. Generally, an increase in the temperature of the solution increases the solubility of a solid solute. A few solid solutes are less soluble in warm solutions. For all gases, solubility decreases as the temperature of the solution increases ${ }^{8}$.

\section{- Pressure}

For gaseous solutes, an increase in pressure increases solubility and a decrease in pressure decrease the solubility. For solids and liquid solutes, changes in pressure have practically no effect on solubility ${ }^{8}$

\section{- Nature of the solute and solvent}

While only 1 gram of lead (II) chloride can be dissolved in 100 grams of water at room temperature, 200 grams of zinc chloride can be dissolved.The great difference in the solubilities of these two substances is the result of differences in their nature ${ }^{8}$.

\section{- Molecular size}

The larger the molecule or the higher its molecular weight the less soluble the substance. Larger molecules are more difficult to surround with solvent molecules in order to 
solvate the substance. In the case of organic compounds the amount of carbon branching will increase the solubility since more branching will reduce the size (or volume) of the molecule and make it easier to solvate the molecules with solvent ${ }^{9}$.

\section{- Polarity}

Generally non-polar solute molecules will dissolve in nonpolar solvents and polar solute molecules will dissolve in polar solvents. The polar solute molecules have a positive and a negative end to the molecule. If the solvent molecule is also polar, then positive ends of solvent molecules will attract negative ends of solute molecules. This is a type of intermolecular force known as dipole-dipole interaction? ${ }^{9}$.

\section{- Polymorphs}

A solid has a rigid form and a definite shape. The shape or habit of a crystal of a given substance may vary but the angles between the faces are always constant. A crystal is made up of atoms, ions, or molecules in a regular geometric arrangement or lattice constantly repeated in three dimensions. This repeating pattern is known as the unit cell. The capacity for a substance to crystallize in more than one crystalline form is polymorphism ${ }^{10}$.

\section{TECHNIQUES OF SOLUBILITY ENHANCEMENT}

There are various techniques available to improve the solubility of poorly soluble drugs. Some of the approaches to improve the solubility are: ${ }^{11}$

\section{$\checkmark$ Physical Modifications \\ - Particle size reduction}

Particle size reduction can be achieved by micronisation and nanosuspension. Each technique utilizes different equipments for reduction of the particle size.

Micronization- The solubility of drug is often intrinsically related to drug particle size. By reducing the particle size, the increased surface area improves the dissolution properties of the drug. Conventional methods ofparticle size reduction, such as comminution and spray drying, rely upon mechanical stress to disaggregate the active compound. The micronisation is used to increase surface area for dissolution ${ }^{12}$.

Nanosuspension- Nanosuspensions are sub-micron colloidal dispersion of pure particles of drug, which are stabilised by surfactants. The advantages offered by nanosuspension is increased dissolution rate is due to larger surface area exposed, while absence of Ostwald ripening is due to the uniform and narrow particle size range obtained, which eliminates the concentration gradient factor. Techniques for the production of nanosuspensions are as follows ${ }^{13}$.

1. Homogenization- The suspension is forced under pressure through a valve that has nano aperture. This causes bubbles of water to form which collapses as they come out of valves. This mechanism cracks the particles. Three types of homogenizers are commonly used such as conventional homogenizers, sonicators, and high shear fluid processors ${ }^{14}$

2. Wet milling- Active drug in the presence of surfactant is defragmented by milling. The nano suspensionapproach has been employed for drugs including tarazepide, atovaquone, amphotericin B, paclitaxel and bupravaquone. All the formulations are in the researchstage. One major concern related to particle size reduction is the eventual conversion of the high-energy polymorph to a low energy crystalline form, which may not be therapeutically active one ${ }^{15}$. Drying ofnano suspensions can be done by lyophilisation or spray drying

Other techniques for reduction of the particle size includes:-

\section{Sonocrystallisation}

Recrystallization of poorly soluble materials using liquid solvents and antisolvents has also been employed successfully to reduce particle size. The novel approach for particle size reduction on the basis of crystallisation by using ultrasound is sonocrystallisation ${ }^{16}$.

\section{Spray drying}

Spray drying is a commonly used method of drying a liquid feed through a hot gas.

Typically, this hot gas is air but sensitive materials such as pharmaceuticals and solvents like ethanol require oxygenfree drying and nitrogen gas is used instead. The liquid feed varies depending on the material being dried and is not limited to food or pharmaceutical products and may be a solution, colloid or a suspension. This process of drying is a one step rapid process and eliminates additional processing $^{17}$. Spray drying of the acid dispersed in acacia solutions resulted in as much as a50\% improvement in solubility of poorly water soluble salicylic acid ${ }^{18}$.

\section{Modification of the crystal habit}

Polymorphism is the ability of an element or compound to crystallize in more then one crystalline form. Different polymorphs of drugs are chemically identical, but they exhibit different physicochemical properties including solubility, melting point, density,texture, stability etc. Broadly polymorphs can be classified as enantiotropes and monotropes based on thermodynamic properties.

\section{Drug dispersion in carriers}

The solid dispersion approach to reduce particle size and therefore increase the dissolution rate and absorption of drugs was first recognised in $1961^{19}$.The term "solid dispersions" refers to the dispersion of one or more active ingredients in an inert carrier in a solid state, frequently prepared by the melting (fusion) method, solvent method, or fusion solvent-method ${ }^{20}$. Novel additional preparation techniques have included rapid precipitation by freeze hydrophilic polymers. using methods such as melt extrusion $^{21}$. The most commonly used hydrophilic carriers for solid dispersions include polyvinylpyrrolidone ${ }^{22}$, polyethylene glycols ${ }^{23}$, Plasdone-S630 ${ }^{24}$. Many times surfactants may drying and using supercritical fluids and spray drying, often in the presence of amorphous also used in the formation of solid dispersion. Surfactants like Tween-80, Docusate sodium, Myrj-52, Pluronic-F68 and Sodium Lauryl Sulphate used ${ }^{24}$. The solubility of etoposide $^{25}$, glyburide ${ }^{26}$, itraconazole ${ }^{27}$, ampelopsin $^{28}$, valdecoxib ${ }^{29}$, celecoxib ${ }^{30}$, and halofantrine ${ }^{31}$ can be improved by solid dispersion using suitable hydrophiliccarriers. The eutectic combination 
ofchloramphenicol/urea ${ }^{32}$ and sulphathiazole/ urea served as examples for the preparation of a poorly soluble drug in a highly water soluble carrier.

\section{Hot melt method}

Sekiguchi and $\mathrm{Obi}^{34}$ used a hot melt method to prepare solid dispersion. Sulphathiazole and urea were melted together and then cooled in an ice bath. Theresultant solid mass was then milled to reduce the particle size. Cooling leads to supersaturation, but dueto solidification the dispersed drug becomes trapped within the carrier matrix. A molecular dispersion can be achieved or not, depends on the degree of supersaturation and rate of cooling used in the process ${ }^{33}$.

\section{Solvent evaporation method}

Tachibana and Nakumara ${ }^{35}$ were the first to dissolve both the drug and the carrier in a common solvent and then evaporate the solvent under vacuum to produce a solid solution. This enabled them to produce a solid solution of the highly lipophilic carotene in the highly water soluble carrier polyvinylpyrrolidone. An important prerequisite for the manufacture of a solid dispersion using the solvent method is that both the drug and the carrier are sufficiently soluble in the solvent ${ }^{33}$. The solvent can be removed by various methods like by spray-drying ${ }^{36}$ or by freeze dying ${ }^{37}$.Temperatures used for solvent evaporation generally lie in the range $23-65{ }^{0} \mathrm{C}^{38}$.

\section{Media Milling}

The nanosuspensions are prepared by using high-shear media mills. The milling chamber charged with milling media, water, drug, and stabilizer is rotated at a very highshear rate under controlled temperatures for several days (at least 2-7 days). The milling medium is composed of glass, Zirconium oxide, or highly cross-linkedpolystyrene resin. High energy shear forces are generated as a result of the impaction of the milling media with the drug resulting into breaking of microparticulate drug to nanosizedparticles ${ }^{68}$.

\section{Hot-melt extrusion}

Melt extrusion was used as a manufacturing tool in the pharmaceutical industry as early as $1971^{39}$. It has been reported that melt extrusion of miscible components results in amorphous solid solution formation, whereas extrusion of an immiscible component leads to amorphous drug dispersed in crystalline excipient ${ }^{40}$.The process has been useful in the preparation of solid dispersions in a single step.

Table 1: Carriers for solid dispersions ${ }^{41}$

\begin{tabular}{|l|l|l|}
\hline S No. & Chemical Class & Examples \\
\hline 1 & Acids & Citric acid, Tartaric acid \\
\hline $\mathbf{2}$ & Sugars & Dextrose, Sucrose, Sorbitol \\
\hline $\mathbf{3}$ & Polymeric Polyvinylpyrrolidone, Materials PEG-4000,Cellulose & PEG-4000,Cellulose \\
\hline $\mathbf{4}$ & Surfactants & $\begin{array}{l}\text { Polyxyethylene Stearate, } \\
\text { Tweens and Spans }\end{array}$ \\
\hline $\mathbf{5}$ & Miscellaneous & Urethase,Urea \\
\hline
\end{tabular}

\section{Complexation}

Complexation is the association between two or more molecules to form a non bonded entity with a well defined stichiometry. Complexation relies on relatively weak forces such as London forces, hydrogen bonding and hydrophobic interactions. There are many types of complexing agents and a partial list can be found in below table2

\section{Staching complexation}

Staching complexes are formed by the overlap of the planar regions of aromatic molecules. Nonpolar moieties tend to be squeezed out of water by the strong hydrogen bonding interactions of water. This causes some molecules to minimize the contact with water by aggregation of their hydrocarbon moieties. This aggregation is favored by large planar non polar regions in the molecule. Stached complexes can be homogeneous or mixed. The former is known as self association and latter as complexation ${ }^{45,46}$. Some compounds that are known to form staching complexes are as Nicotinamide[5456], Anthracene, Pyrene, Methylene blue, Benzoic acid, Salicylic acid, Ferulic acid, Gentisic acid, Purine, Theobromine, Caffeine, and Naphthalene etc.
Inclusion complexes are formed by the insertion of the non polar molecule or the non polar region of one molecule (known as guest) into the cavity of another molecule or group of molecules (known as host). The major structural requirement for inclusion complexation is a snug fit of the guest into the cavity of host molecule. The cavity of host must be large enough to accommodate the guest and small enough to eliminate water, so that the total contact between the water and the nonpolar regions of the host and the guest is reduced ${ }^{42,44}$.

Table 2: List of complexing agent ${ }^{47}$

\begin{tabular}{|c|l|l|}
\hline SN. & Types & Examples \\
\hline 1 & Inorganic & $\mathrm{I}_{\mathrm{B}^{-}}$ \\
\hline 2 & Coordination & Hexaminecobalt(III) chloride \\
\hline 3 & Chelates & EDTA, EGTA \\
\hline 4 & Metal & Olefin Ferrocene \\
\hline 5 & Inclusion & Cyclodextrins, Choleic acid \\
\hline 6 & $\begin{array}{l}\text { Molecular } \\
\text { Complexes }\end{array}$ & Polymers \\
\hline
\end{tabular}

\section{Inclusion complexation}




\section{Solubilization by surfactants}

Surfactants are molecules with distinct polar and non polar regions. Most surfactants consist of a hydrocarbon segment connected to a polar group. The polar group can be anionic, cationic, zwitter ionic or non ionic ${ }^{48}$. When small polar molecules are added they can accumulate in the hydrophobic core of the micelles. This process of solubilization is very important in industrial and biological processes. The presence of surfactants may lower the surface tension and increase the solubility of the drug within an organic solvent ${ }^{49}$.

\section{Microemulsion}

The term microemulsion was first used by Jack $H$. Shulman in 1959. A microemulsion is a four component system composed of external phase, internal phase, surfactant and cosurfactant. The addition of surfactant, which is predominately soluble in the internal phase unlike the co-surfactant, results in the formation of an optically clear, isotropic, thermodynamically stable emulsion. It is termed as microemulsion because of the internal or dispersed phase is $<0.1 \mu$ droplet diameter ${ }^{50}$.

\section{Chemical Modifications}

For organic solutes that are ionizable, changing the $\mathrm{pH}$ of the system may be simplest and most effective means of increasing aqueous solubility. Under the proper conditions, the solubility of an ionizable drug can increase exponentially by adjusting the $\mathrm{pH}$ of the solution. A drug that can be efficiently solubilized by $\mathrm{pH}$ control should be either weak acid with a low pKa or a weak base with a high pKa. Similar to the lack of effect of heat on the solubility of non-polar substances, there is little effect of $\mathrm{pH}$ on non ionizable substances ${ }^{51}$.

\section{Co-crystallisation}

The new approach available for the enhancement of drug solubility is through the application of the co-crystals, it is also referred as molecular complexes. If the solvent is an integral part of the network structure and forms at least two component crystals, then it may be termed as cocrystal. If the solvent does not participate directly in the network itself, as in open framework structures, then it is termed as clathrate (inclusion complex). A co-crystal may be defined as a crystalline material that consists of two or more molecular (and electrically neutral) species held together by non-covalent forces. Co-crystals are more stable, particularly as the co-crystallizing agents are solids at room temperature ${ }^{52}$.

\section{Co-solvency}

The solubilisation of drugs in co-solvents is a technique for improving the solubility of poorly soluble drug ${ }^{53}$. It is well-known that the addition of an organic co solvent to water can dramatically change the solubility of drugs. Most co solvents have hydrogen bond donor and/or acceptor groups as well as small hydrocarbon regions. Their hydrophilic hydrogen bonding groups ensure water miscibility, while their hydrophobic hydrocarbon regions interfere with waters hydrogen bonding network, reducing the overall intermolecular attraction of water. By (c) 2011, JDDT. All Rights Reserved disrupting waters self-association, co solvents reduce waters ability to squeeze out non-polar, hydrophobic compounds, thus increasing solubility. A different perspective is that by simply making the polar water environment more non polar like the solute, cosolvents facilitate solubilization. Solubility enhancement as high as 500 -fold is achieved using 20\% 2-pyrrolidone ${ }^{54}$.

\section{Molecular encapsulation with cyclodextrins}

The beta- and gamma- cyclodextrins and several of their derivatives are unique in having the ability to form molecular inclusion complexes with hydrophobic drugs having poor aqueous solubility. These bucket shaped oligosaccharides produced from starch are versatile in having a hydrophobic cavity of size suitable enough to accommodate the lipophilic drug as guests; the outside of the host molecule is relatively hydrophilic. Thus the molecularly encapsulated drug has greatly improved aqueous solubility and dissolution rate. There are several examples of drugs with improved bioavailability due to such phenomenon- thiazide diuretics, barbiturates and a number of NSAIDs ${ }^{55}$.

\section{Solubilizing agents}

The solubility of poorly soluble drug can also be improved by various solubilizing materials. PEG 400 is improving the solubility of hydrochlorthiazide ${ }^{56}$. Modified gum karaya (MGK), a recently developed excipient was evaluated as carrier for dissolution enhancement of poorly soluble drug, nimodipine. The aqueous solubility of the antimalarial agent halofantrine is increased by addition of caffeine and nicotinamide ${ }^{57}$.

\section{Nanotechnology approaches}

Nanotechnology will be used to improve drugs that currently have poor solubility. Nanotechnology refers broadly to the study and use of materials and structures at the nanoscale level of approximately 100 nanometers (nm) or less. For many new chemical entities of very low solubility, oral bioavailability enhancement by micronisation is not sufficient because micronized product has the tendency of agglomeration, whichleads to decreased effective surface area for dissolution and the next step taken was Nanonisation ${ }^{58}$.

\section{Nanocrystal}

A nanocrystal is a crystalline material with dimensions measured in nanometers; a nano particle with structure that is mostly crystalline. The nano-crystallization is defined as a way of diminishing drug particles to the size range of 11000 nanometers. Nano-crystallization is thought to be an universal method that can be applied to any drug ${ }^{59}$. There are two distinct methods used for producing nanocrystals; 'bottom-up' and 'top-down' development ${ }^{60}$. The top-down methods (i.e. Milling and High pressure homogenization) start milling down from macroscopic level, e.g. from a powder that is micron sized. In bottom-up methods (i.e. Precipitation and Cryo-vacuum method), nano scale materials are chemically composed from atomic and molecular components. 
Nano scale particles can be produced by wet-milling process $^{61}$. In ball mills, particle size reduction is achieved by using both impact and attrition forces. The most common models are a tumbling ball mill and a stirred media mill. One problem of this method is the degradation of mill surfaces and subsequent suspension contamination.

\section{High pressure homogenization}

In high pressure homogenization, an aqueous dispersion of the crystalline drug particles is passed with high pressure through a narrow homogenization gap with a very high velocity ${ }^{62}$. Homogenisation can be performed in water (Disso Cubes) or alternatively innon-aqueous media or water-reduced media (Nanopure) . The particles are disintegrated by cavitation and shear forces. The static pressure exerted on the liquid causes the liquid to boil forming gas bubbles ${ }^{63}$. The particle size obtained during the homogenization process depends primarily on the nature of the drug, the pressure applied and the number of homogenization cycles.

\section{Precipitation}

In the precipitation method a dilute solution is first produced by dissolving the substance in a solvent where its dissolution is good $^{64}$. The solution with the drug is then injected into water, which acts as a bad solvent. At the time of injection, the water has to be stirred efficiently so that the substance will precipitate as nano-crystals. Nanocrystals can be removed from the solution by filtering and then dried in air.

\section{NanoMorph}

The Nano Morph technology is to convert drug substances with low water-solubility from a coarse crystalline state into amorphous nano particles. A suspension of drug substance in solvent is fed into a chamber, where it is rapidly mixed with another solvent. Immediately the drug substance suspension is converted into a true molecular solution. The admixture of an aqueous solution of a polymer induces precipitation of the drug substance. The polymer keeps the drug substance particles in their nanoparticulatestate and prevents them from aggregation or growth. Water redispersable dry powders can be obtained from the nanosized dispersion by conventional methods, e.g. nspray-drying. Nanotechnology approaches to improve the solubility of hydrophobic drugs shown in table.

Table 4: Nanotechnology approaches to improve the solubility of hydrophobic drugs ${ }^{65,66}$

\begin{tabular}{|l|l|l|}
\hline Company & $\begin{array}{l}\text { Nanoparticulate } \\
\text { Technologies }\end{array}$ & Description \\
\hline Elan & Nano Crystal & $\begin{array}{l}\text { Nano crystal drug particles }(<1000 \mathrm{~nm}) \text { produced by wetmilling and stabilised } \\
\text { against agglomeration via surface adsorption of stabilizers applied to NMEs eg. } \\
\text { Reformulation of existing drugs eg. Sirolimus }\end{array}$ \\
\hline BioSante & CAP & $\begin{array}{l}\text { Calcium-phosphate based nanoparticles: for improved oral bioavailability of } \\
\text { hormones/proteins such as insulin }\end{array}$ \\
\hline
\end{tabular}

\section{Hydrotropy}

Hydrotropy is a solubilization phenomenon whereby addition of large amount of a second solute results in an increase in the aqueous solubility of another solute. Concentrated aqueous hydrotropic solutions of sodium benzoate, sodium salicylate, urea, nicotinamide, sodium citrate and sodium acetate have been observed to enhance the aqueous solubilities of many poorly water soluble drugs ${ }^{67}$.

\section{Micellar solubilization}

The use of surfactants to improve the dissolution performance of poorly soluble drug products is probably the basic, primary, and the oldest method. Surfactants reduce surface tension and improve the dissolution of lipophilic drugs in aqueous medium. They are also used to stabilise drug suspensions. When the concentration of surfactants exceeds their critical micelle concentration (CMC, which is in the range of $0.05-0.10 \%$ formost surfactants),micelle formation occurs which entrap the drugs within the micelles. This is known as micellization and generally results in enhanced solubility of poorly soluble drugs. Surfactant also improves wetting of solids and increases the rate of disintegration of solid into finer particles ${ }^{69}$. Commonly used non-ionic surfactants include polysorbates, polyoxyethylated castor oil, polyoxyethylated glycerides, lauroyl macroglycerides, and mono- and di-fatty acid esters of low molecular weight polyethylene glycols. Surfactants are also often used to stabilize microemulsions and suspensions into which drugs are dissolved ${ }^{70,71}$.

Examples of poorly soluble compounds that useMicellar solubilization are antidiabetic drugs, gliclazide, glyburide,glimepiride, glipizide, repaglinide, pioglitazone, and rosiglitazone $\mathrm{e}^{72}$.

\section{Supercritical fluid (scf) process}

Another novel nanosizing and solubilisation technology whose application has increased in recent years is particle size reduction via supercritical fluid (SCF) processes. Supercritical fluids are fluids whose temperature and pressur eare greater than its critical temperature $(\mathrm{Tc})$ and critical pressure (Tp), allowing it to assume the properties of both a liquid and a gas. At near-critical temperatures, SCFs, are highly compressible allowing moderate changes in pressure to greatly alter the density and mass transport characteristics of the fluid that largely determine its solvent power. Once the drug particles are solubilised within the SCF (usually carbon dioxide), theymay be recrystallised at greatly reduced particle sizes. The flexibility and precision offered by SCF processes allows micronisation of drug particles within narrow ranges of particle size, often to submicron levels. Current SCF processes have demonstrated the ability to create nanoparticulate suspensions of particles $5-2,000 \mathrm{~nm}$ in diameter. Several pharmaceutical companies, such as 
Nektar Therapeutics and Lavipharm, are specializing in particle engineering via SCF technologies for particle size reduction and solubility enhancement. Several methods of SCF processing have been developed to address individual aspects of these shortcomings, such as precipitation with compressed antisolvent process (PCA), solution enhanced dispersion by SCF (SEDS), supercritical antisolvent processes (SAS), rapid expansion of supercritical solutions (RESS), gas anti solvent recrystallization (GAS), and aerosol supercritical extraction system (ASES) ${ }^{73}$.

\section{CONCLUSION}

Dissolution of drug is the rate determining step for oral absorption of the poorly water soluble drugs and solubility is the basic requirement for the absorption of the drug from
GIT. The various techniques described above alone or in combination can be used to enhance the solubility of the drugs. Proper selection of solubility enhancement method is the key to ensure the goals of a good formulation like good oral bioavailability, reduce frequency of dosing and better patient compliance combined with a low cost of production. Selection of method for solubility enhancement depends upon drug characteristics like solubility, chemical nature, melting point, absorption site, physical nature, pharmacokinetic behavior and so forth, dosage form requirement like tablet or capsule formulation, strength, immediate, or modified release and so forth, and regulatory requirements like maximum daily dose of any excipients and/or drug, approved excipients, analytical accuracy and so forth.

\section{REFERENCES}

1. Rinaki E, Valsami G, and Macheras P; Quantitative Biopharmacuetics Classification System; the central role of dose/solubility ratio. Pharm. Res. 2003; 20:1917.

2. Youn YS, JY Tung. Shoh, SD Yoo, K C Lee. Improved intestinal delivery of salmon calcitonin by Lys18-amine specific PEGylation: Stability, permeability, pharmacokinetic behavior and in vivo hypocalcemic efficacy. J. Contr. Rel 2006;144::334-42

3. Sugawara M, S Kadomura, H Xin, Y. Takekuma, N Khori, K Miyazaki. The use of an in vitro dissolution andabsorption system to evaluate oral absorption of two weakbases in $\mathrm{pH}$ independent controlled-release formulations.Eur. J. Pharm. 2005, Sci.26: 1-8.

4. Improving solubility \& permeability in drugcandidates. Conference: 23rd \& 24th June,2005, Pre-conference workshop: 22nd June2005, Thistle Marble Arch, London, UK.

5. James K., Solubility and related properties.28: Marcel Dekker Inc., Newyork, 986, $127-395$.

6. http://www.cop.ufl.edu/safezone/prokai/pha5100/pha5110.htm

7. http://www.chem.lsu.edu/lucid/tutorials/tutorials.html

8. shttp://www.sciencebyjones.comm

9. http://www.chem.lsu.edu/lucid/tutorials/tutorials.html

10. Singhal D, Curatolo W.Drug polymorphismanddosage form design a practical perspective, Adv. Drug. Deliv. Rev., 2004; 56, 335 347.

11. Pinnamaneni S., Das N.G., DasS.K.Formulation approaches for orallyadministered poorly soluble drugs. Pharmazie,2002; 57:291 300

12. Chaumeil J.C.Micronisation: a method of improving the bioavailability of poorlysolubledrugs, Methods and Findings inExperimental and Clinical Pharmacology,1998;20:211-215.

13. Nanosuspension drug delivery Technologyand application Nanotech - Express PharmaPulse.htm,

14. Medical Design Technology online atwww.mdtmag.com or Microfluidics athttp://www.microfluidicscorp.com

15. M.E.Aulton .Pharmaceutics, The science ofdosage form design, 2nd edition, ChurchillLivingstone, London, 2002;113-252.

16. Banga Sheere, Chawala Garima, BansalArvind K .New Trends in the Crystallisationof Active Pharmaceutical Ingredients,Businessbriefing Pharmagenerics 2004;70-74.

17. Spray drying From Wikipedia, the freeencyclopedia. Retrieved fromhttp://en.wikipedia.org

18. Kawashima Y, Saito M, Takenaka H, Improvement of solubility and dissolution rateof poorly water-soluble salicylic acid by aspraydrying technique. J. Pharm. Pharmacol.1975, 27:1-5.

19. Sekiguchi K,Obi N. Studies on absorption of eutectic mixtures. I. A comparison of thebehavior of eutectic mixtures ofsulphathiazole and that of ordinarysulphathiazole in man, Chem. Pharm. Bull.,1961;9: 866-872.

20. Chiou W L, Riegelman S. Pharmaceuticalapplications of solid dispersion systems, J.Pharm. Sci. 1971; 60: 1281-1302.

21. Forster A, Hempenstall J, Rades T. Characterization of glass solutions of poorlywater-soluble drugs produced by meltextrusion with hydrophilic amorphous polymers, J. Pharm. Pharmacol., 2001, 53:303-315.
22. Ambike AA, Mahadik KR, Paradkar A. Stability study of amorphous valdecoxib. Int. J.Pharm. 2004, 282: 151-162.

23. Doshi DH, Ravis WR, Betageri GV. Carbamazepine and polyethylene glycol soliddispersion preparation, invitro dissolution, andcharacterization. Drug Dev Ind Pharm., 1967,23: 1167-1176.

24. Ghebremeskel Alazar N, Vemavarapu Chandra, Lodaya Mayur. Use of surfactantsas plasticizers in preparing solid dispersions ofpoorly soluble API: Selection of polymer-surfactant combinations using solubilityparameters and testing the processabilitInternational Journal of Pharmaceutics2007,328: 119-129.

25. Shah Jaymin C, Che Jivn R, Diana Chow,Preformulation study of etoposide: II.Increased solubility and dissolution rate bysolid-solid dispersions, InternationalJournal of Pharmaceutics 1995, 113:103111 .

26. Betageri G V, Makarla K R. Enhancement ofdissolution of glyburide by solid dispersionand lyophilization techniques. InternationalJournal of Pharmaceutics, 1995, 126: 155-160.

27. Jae-Young Jung, Sun Dong Yoo, Sang-HeonLee, Kye-Hyun Kim, Doo-Sun Yoon, Kyu-Hyun Lee. Enhanced solubility and dissolution rate of itraconazole by a solidDispersion technique. International Journal ofPharmaceutics, 1999, 187:209-218.

28. Li-Ping Ruan, Bo-Yang Yu, Guang-Miao Fu,Dan-ni Zh. Improving the solubility ofampelopsin by solid dispersions and inclusioncomplexes.Journal of Pharmaceutical andBiomedical Analysis 2005, 38: 457-464.

29. Aftab Modi and Pralhad Tayade,Enhancement of Dissolution Profile by SolidDispersion (Kneading) Technique, AAPSPharmSciTech 2006, 7 (3) Article 68.

30. Gupta Piyush , Kakumanu Vasu Kumar ,Bansal Arvind K.Stability and Solubility ofCelecoxib-PVP Amorphous Dispersions: A Molecular Perspective, PharmaceuticalResearch 2004, 21:17621769.

31. Ahmad M. Abdul-Fattah, Hridaya N.Bhargava.Preparation and in vitro evaluationof solid dispersions of Halofantrine.International Journal of Pharmaceutics 2002,235: 17-33.

32. Sekiguchi K, Obi N, Ueda Y. Studies onabsorption of eutectic mixtures. II. Absorptionof fused conglomerates of chloramphenicoland urea in rabbits, Chem. Pharm. Bull. 1964;12: 134-144.

33. Christian Leuner, Jennifer Dressman.Improving drug solubility for oral deliveryusing solid dispersions. European Journal ofPharmaceutics and Biopharmaceutics, 2000,50:47-60.

34. Sekiguchi K,Obi N. Studies on absorption ofeutectic mixtures. I. A comparison of thebehavior of eutectic mixtures ofsulphathiazole and that of ordinarysulphathiazole in man, Chem. Pharm. Bull., 1961;9: 866-872.

35. Tachibana T, Nakamura A. A method for preparing an aqueous colloidal dispersion of organic materials by using watersolublepolymers: dispersion of beta-carotene bypolyvinylpyrrolidone, Kolloid-Z. Polym.1965, 203: 130-133. 36. Lo W Y ,Law S L.Dissolution behavior ofgriseofulvin solid dispersions usingpolyethylene glycol, talc, and theircombination as dispersion carriers, Drug Dev.Ind. Pharm. 1996, 22: 231-236. 
Thakur et al

Journal of Drug Delivery \& Therapeutics; 2013, 3(2), 215-221

37. Betageri G V, Makarla K R. Enhancement ofdissolution of glyburide by solid dispersionand lyophilization techniques. Int. J. Pharm1995, 126: 155-160.

38. Kearney A S, Gabriel D L, Mehta S C,Radebaugh G W. Effect ofpolyvinylpyrrolidone on the crystallinity anddissolution rate of solid dispersions of theantiin ammatory Ci-987. Int. J. Pharm 1994,104: 169-174.

39. el-Egakey MA, Soliva M, Speise P. Hot extruded dosage forms. Pharm Acta Helv. 1971, 46: 31-52.

40. Breitenbach J. Melt extrusion: from process to drug delivery technology. Eur J Pharm Biopharm 2002, 54:107-117

41. Chiou W L, Riegelman S. Pharmaceutical applications of solid dispersion systems, J.Pharm. Sci. 1971; 60: 1281-1302

42. Joon Woo Park, Kinetics and Mechanism of Cyclodextrin Inclusion Complexation Incorporating Bidirectional Inclusion and Formation of Orientational Isomers, J. Phys. Chem. B, ASAP Article, www.aaps.org

43. Kaneto Uekama, Fumitoshi Hirayama, and Tetsumi Irie. Cyclodextrin Drug Carrier Systems, Chem. Rev. 1998, 98: 2045 2076.

44. Singala Anil K, Garg Alka, Aggarwal Deepika. Paclitaxel and its formulations. International Journal of Pharmaceutics 2002, 235: 179192.

45. Sanghvi Ritesh, Daniel Evans, Samuel H. Yalkowsky, Stacking complexation by nicotinamide: A useful way of enhancing drug solubility. International Journal of Pharmaceutics, 2007, 336: 35-41. 46. Higuchi T., Kristiansen H. Binding specificities between small organic solutes in aqueous solutions: classification of some solutes into two groups according to binding tendencies. J. Pharm. Sci. 1970, 59:1601-1608..

47. http://www.cop.ufl.edu/safezone/prokai/pha51

48. Swarbrick J., Boylan J.C. Encyclopedia of Pharmaceutical Technology; 2nd edn, 2002,3:2458-2479.00/pha5110.htm

49. Michael Hite, Lead Research Associate, Stephen Turner, Oral Delivery of Poorly Drugs 400, Pharmaceutical Manufacturing and Packing Sourcer Summer '03 issue. Samedan Ltd. 2003.

50. Tenjarla SN. Microemulsions: An overview and pharmaceutical applications. Critical Reviews TM in Therapeutic Drug Carrier Systems, 1999, 16:461-521.

51. Solutions and Solubility. Adam M. Persky and Jeffrey A. Hughes, University of Florida, College of Pharmacy, Gainesville, FL 32610, USA

52. Aakeröy C B. Crystal engineering: Strategies andArchitectures, Acta Cryst. 1997, B53:569-586.

53. Amin K, Dannenfelser R M, Zielinski J, Wang B. Lyophilization of polyethylene glycol mixtures. J. Pharm. Sci. 2004, 93:2244-2249.

54. Jain Parijat, Yalkowsky Samuel H. Solubilization of poorly soluble compounds using 2-pyrrolidone.International Journal of Pharmaceutics 2007.

55. Rao M.G. et al. Preparation and evaluation of solid dispersion of naproxen. Indian J. Pharm. Sci., 2005, 67: 26-29.

56. Vervaet C, Remon JP. Bioavailability of hydrochlorothiazide from pellets, made by extrusion/spheronisation, containing 1993, 41:737-740. polyethylene glycol 400 as a dissolution enhancer, Pharm Res. 1997, 14:1644-1646.

57. Lee-Yong Lim, Mei-Lin Go, Caffeine and nicotinamide enhances the aqueous solubilityof the antimalarial agent halofantrine. European Journal of Pharmaceutical Sciences, 2000, 10: 17-28.

58. Keck CM, Müller RH. Drug nanocrystals of poorly soluble drugs produced by high pressure homogenisation. Eur J Pharm Biopharm. 2006, 62: 3-16.

59. Radtke M.Pure dug nanoparticles for formulation of poorly soluble drugs. New Drugs, 2001, 3: 62-68.

60. Mazzola L. Commercializing nanotechnology. Nat Biotechnol, 2003, 10:1137-1143.

61. Kondo N, Iwao T, Masuda H, Yamanouchi K, Ishihara Y, Yamada N, Haga T, Ogawa Y, Yokoyama K. Improved oral absorption of a poorly water-soluble drug, HO-221, by wetbead milling producing particles in submicron region. Chem Pharm Bull,

62. RH, Becker R, Kruss B, Peters K.Pharmaceutical nanosuspensions for medicament administration as systems with increased saturation solubility and rate of solution, 1999, US Patent 5858410.

63. Hecq J, Deleers M, Fanara D, and Vranckx H, Amighi K. Preparation and characterization of nanocrystals for solubility and dissolution rate enhancement of nifedipine. Int J Pharm, 2005, 299: 167-177.

64. Chung H-R, Kwon E, Oikawa H, Kasai H, Nakanishi H.Effect of solvent on organic nanocrystal growth using the reprecipitation method. J Cryst Growth, 2006, 2:459- 463.

65. NanoMorph technology for Amorphous Nanoparticles, www.soliqs.com

66. Roghieh Saffie-Siebert, Dr Jill Ogden and Dr Mark ParryBillings. Nanotechnology approaches to solving the problems of poorly water-soluble drugs, Drug Discovery World Summer, 2005, 71-76.

67. Maheshwari R.K. A novel application of hydroscopic solubilization in Spectrometric.V. B. Patravale, A. A. Date, and R. M. Kulkarni, "Nanosuspensions:a promising drug delivery strategy," Journal of Pharmacy and Pharmacology, vol. 56, no. 7, pp. 827-840, 2004.

69 K. H. Edward and D. Li, "Solubility," in Drug Like Properties: Concept, Structure, Design and Methods, from ADME to Toxicity Optimization, p. 56, Elsevier, 2008.

70 A. Martin, Physical Pharmacy, Willaims and Wilkins, Baltimore, Md, USA, 4th edition, 1993.

71 C. D. Rangel-Yagui, A. Pessoa, and L. C. Tavares, "Micellar solubilisation of drugs," Journal of Pharmacy and Pharmaceutical Sciences, vol. 8, no. 2, pp. 147-163, 2005.

72 C. H. Hsu, Z. Cui, R. J. Mumper, and M. Jay, "Micellar solubilisation of some poorly soluble antidiabetic drugs," AAPS PharmSciTech, vol. 9, no. 2, pp. 939-943, 2008.

73 G. Sunkara and U. B. Kompella, "Drug delivery applications of supercritical fluid technology," Drug Delivery Technology, vol. 2, pp. 44-50, 2002. 